\title{
RANDOM LABELLED TREES AND THEIR BRANCHING NETWORKS
}

\author{
G. R. GRIMMETT \\ (Rcieived 2 January; revised 20 March 1980) \\ Communicated by W. S. Wallis
}

\begin{abstract}
A random rooted labelled treo un $n$ vertices has asymptotically the same shape as a branching-type process, in which each generatiun of a branching process with Poisson family sizes, parameter one, is supplemented by a single additional member added at random to one of the families in that generation. In this note we use this probabilistic representation to deduce the asymptotic distribution of the distance from the root to the nearest endvertex other than itself.
\end{abstract}

1980 . Wathematics subject clasification (Amer. Math. Soc.): primary $05 \mathrm{C} 05$ : secondary $60 \mathrm{~J} 80$.

Keywords: random tree, randwn graph, branching process.

\section{Introduction}

There are $n^{n-2}$ different trees on the labelled vertex set $\left\{v_{1}, v_{2}, \ldots, v_{n}\right\}$. Let $T_{n}$ denote a randomly chosen member of this collection, each member having equal probability of being chosen. Meir and Moon have explored many properties of $T_{n}$ when $n$ is large; their methods are largely enumerative and analytical and make considerable use of the exponential generating function

$$
y=\sum_{n} n^{n-2} x^{n} /(n-1) !
$$

of the numbers of labelled trees. It is the purpose of this note to exploit a probabilistic representation of $T_{n}$ in terms of branching processes in order to solve a problem concerning the shortest distance between a specified vertex and the endvertices of $T_{n}$ other than this vertex.

A random walk about the vertices of $T_{n}$ is a sequence $V_{0}, V_{1}, V_{2}, \ldots$ of vertices, possibly with repetition, randomly chosen according to the rule that $V_{k+1}$ is picked uniformly at random from the collection of vertices of $T_{n}$ which are adjacent to $V_{k} ; V_{0}$ 
is a specified starting point. Conditional upon $T_{n}$ being given, $\left\{V_{i}: i=0,1, \ldots\right\}$ is a Markov chain; it is easy to check that it has stationary distribution $\left\{\pi_{i}: i=1,2, \ldots, n\right\}$ given by

$$
\pi_{i}=d_{i}(2(n-1))^{-1} \quad(i=1,2, \ldots, n),
$$

where $d_{i}$ is the degree of the vertex $v_{i}$ in $T_{n}$; a similar result holds for random walks about the vertex set of any finite undirected graph. Meir and Moon have been concerned with certain first passage times associated with the chain. For example, Moon (1973) shows that the time $v_{n}$ of the first visit to $r_{2}$ starting from $v_{1}$, given formally by

$$
v_{n}=\min \left\{m: V_{m}=v_{2}\right\} \quad \text { given } V_{0}=v_{1},
$$

satisfies

$$
E\left(v_{n}\right) \sim\left(\frac{1}{2} \pi n^{3}\right)^{\frac{1}{2}} .
$$

Also, Meir and Moon (1975) show that the time $\lambda_{n}$ of the earliest visit to an endvertex of $T_{n}$ after the commencement of the walk, given by

$$
\lambda_{n}=\min \left\{m \geqslant 1: V_{m} \text { is an endvertex }\right\} \text { given } V_{0}=v_{1},
$$

satisfies

$$
P\left(\hat{\lambda}_{n}=k\right) \rightarrow k p^{2} q^{k-1}, \quad \text { where } p=1-q=e^{-1},
$$

and

$$
E\left(\hat{\lambda}_{n}\right) \rightarrow 2 e-1
$$

Related work on climbing random trees is described in Moon (1976) and Meir and Moon (1978a). Result (1) contrasts strongly with an earlier result of Meir and Moon (1970, 1978b), which asserts that the number $\gamma_{n}$ of edges in the unique path of $T_{n}$ joining vertices $v_{1}$ and $v_{2}$ has mean value

$$
E\left(\gamma_{n}\right) \sim\left(\frac{1}{2} \pi n\right)^{\frac{1}{2}}
$$

Here, we answer the corresponding question to result (2): what is the limiting distribution as $n \rightarrow \infty$ of the distance between $v_{1}$ and the nearest endvertex of $T_{n}$ other than $v_{1}$ ? In Section 2 we describe an enumerative approach to the problem, in the spirit of Rényi and Szekeres (1967), who used techniques of complex analysis to answer a similar question. In Section 3 we show that, for large $n, T_{n}$ has asymptotically the same stochastic shape as a random process defined in terms of branching processes. This observation enables us to use easy recursive arguments to solve the problem in question. Other problems may be susceptible to solution by the same method. 
Similar combinatorial and probabilistic arguments may be used to find the asymptotic distribution of the distance from $v_{1}$ to the nearest endvertex, including $v_{1}$ if it has degree one.

See Grimmett (1980) for a review of the theory of random trees and other random graphs.

\section{A combinatorial approach}

Let $T$ be a labelled $n$-1ree with vertices $v_{1}, v_{2}, \ldots, v_{n}$, and let $d(T)$ be the number of edges in the shortest path from $v_{1}$ to an endvertex of $T$, other than $v_{1}$ if it has degree one. Let $T(k, n)=|\{T: d(T) \geqslant k\}|$ be the number of $n$-trees with $d(T) \geqslant k$, and let

$$
G_{k}(x)=\sum_{n=1}^{x} T(k, n) x^{n} /(n-1) !
$$

be the exponential generating function of the $T(k, n)$ for fixed $k$. It is clear that $T(0, n)=n^{n-2}$, and it follows that $G_{0}(x)$ satisfies

$$
G_{0}(x)=x \exp \left(G_{0}(x)\right) .
$$

Furthermore, if $n, k \geqslant 1$.

$$
\begin{aligned}
T(k, n)=\sum_{r=1}^{n-1}\left(\begin{array}{c}
n-1 \\
r
\end{array}\right) & \sum_{\mathrm{a}}\left(\begin{array}{c}
n-r-1 \\
a_{1}-1, \ldots, a_{\mathrm{r}}-1
\end{array}\right) \\
& \times T\left(k-1, a_{1}\right) T\left(k-1, a_{2}\right) \ldots T\left(k-1, a_{r}\right),
\end{aligned}
$$

where $\sum_{\mathrm{a}}$ sums over all sequences $\left\{a_{1}, a_{2}, \ldots, a_{r}\right\}$ with sum $n-1$. This holds because $d(T) \geqslant k$ if and only if each vertex $v$ adjacent to $v_{1}$ is at least distance $k-1$ from the nearest endvertex other than $v$ itself in the subtree of $T$ comprising all vertices including $v$ whose unique path to $v_{1}$ passes through $v$. Multiply (4) by $x^{n} /(n-1)$ ! and sum over $n$ to obtain

$$
G_{k}(x)=x\left(\exp \left(G_{k-1}(x)\right)-1\right)
$$

which is a functional recurrence relation for the $G_{k}$ subject to the condition

$$
G_{0}(x)=\sum_{n} n^{n-2} x^{n} /(n-1) !
$$

It may be possible to use these relations to determine the asymptotic probabilities

$$
\lim _{n \rightarrow x} T(k, n) n^{2-n}
$$

that a large random tree $T_{n}$ has $d\left(T_{n}\right) \geqslant k$. Rényi and Szekeres (1967) have been able to do this in a similar case; however, it seems more natural to proceed by probabilistic techniques. 


\section{A branching-type process}

Let $\left\{X_{i}: i \geqslant 0\right\}$ be the sizes of the generations in a branching process in which $X_{0}=1$ and each family size has the Poisson distribution with parameter $\lambda$. The total progeny

$$
N=\sum_{i=0}^{x} X_{i}
$$

is almost surely finite if and only if $\lambda \leqslant 1$. The graphical representation of the process is a tree with $N$ vertices which is similar in appearance to a random labelled $N$-tree in the following sense. Consider the random tree $T_{n}$ rooted at $v_{1}$. The $k$ th stratum of $T_{n}$ is the collection of vertices of $T_{n}$ which are exactly distance $k$ from $v_{1}$. Let $Z(k, n)$ be the size of the $k$ th stratum.

THEOREM 1 (Kolchin (1977)). The sequence $\{Z(i, n): 0: \because i<n\}$ has the same joint distribution as the sequence $\left\{X_{i}: 0 \leqslant i<x\right\}$ conditional upon the ectent $\{N=n\}$. That is,

$$
P\left(Z(i, n)=r_{i}, 0 \leqslant i<n\right)=P\left(X_{i}=r_{i}, 0 \leqslant i<x \mid N=n\right) .
$$

Kennedy (1956) has studied asymptotic properties of a branching process conditioned upon the value of the total progeny. His results imply that the asymptotic distribution are independent of $i$; henceforth we assume that $i=1$. Furthermore he shows the following

THEOREM 2 (Kennedy (1975)). If $i_{1}<i_{2}<\ldots<i_{k}$ then

$$
\lim _{n \rightarrow x} P\left(X_{i_{j}}=r_{j}, j=1,2, \ldots, k \mid N=n\right)=r_{k} P\left(X_{i j}=r_{j}, j=1,2, \ldots, k\right)
$$

These two theorems contain information about the likily shape of a random tree as $n \rightarrow \infty$. When suitably corrected, Kennedy's Theorem 3 contains as a special case a third solution to the problem, independently solved twice already by Rényi and Szekeres (1967) and Stepanov (1969), of determining the asymptotic distribution of the height of a random tree.

It is not difficult to deduce that the asymptotic form of $T_{n}$ is the same as that of branching type process $T$ defined as follows. A population is constructed recursively according to generation number by the stochastic rules:

(a) The zeroth generation contains one member;

(b) For $k \geqslant 0$, the $(k+1)$ th generation $A_{k+1}$ is the union of the families of descendants of the members of the $k$ th generation $A_{k}$, together with one additional member which is allocated at random to one of these families, each of 
the $\left|A_{k}\right|$ families having equal probability of being chosen. Before the allocation of this supplementary member, all family sizes are independent of each other and the past, and are Poisson distributed with parameter one. Hence, for example, the size $Y_{k}$ of the $k$ th generation satisfies

$$
Y_{k+1}=1+F_{1}+F_{2}+\ldots+F_{Y^{\prime}}
$$

where $\left\{F_{i}: i \geqslant 1\right\}$ is a collection of independent Poisson variables; the process can never become extinct. Realizations may be represented graphically as trees rooted at the initial member, with edges joining father-son pairs.

The next theorem concerns the asymptotic shape of $T_{n}$ for large $n$. Let $k$ be a positive integer. Draw the subtree of $T_{n}$ comprising the vertices of $T_{n}$ which are in one of the first $k$ strata only. Within any family, order the vertices in, say, increasing lexicographic order, and then delete all labels except that of the root; call the resulting random tree $T_{n}^{k}$.

THEOREM 3. As $n \rightarrow$ y the characteristics of $T_{n}^{k}$ become indistinguishable in distribution from the corresponding characteristics of the tree representation of the first $k$ generations of the hranching type process $T$ described above.

Thus the probabilities of events such as $\left\{d\left(T_{n}\right) \geqslant k\right\}$, which are describable in terms of the first $l$ strata of $T_{n}$ for some $l$, approach as $n \rightarrow \infty$ the probabilities that the corresponding events occur in $T$. That is, instead of calculating $P\left(d\left(T_{n}\right) \geqslant k\right)$ and letting $n \rightarrow \infty$, we may first let $n \rightarrow \infty$ and then calculate the limit probability directly.

The next theorems are corollaries.

THEOREM 4. The number $N(k, n)$ of endvertices of $T_{n}$ in the kth stratum has probability generating function

$$
H_{k, n}(x)=\left.E\left(x^{N(k, n)}\right) \rightarrow \frac{d}{d y} f_{k}(y)\right|_{y=y_{0}},
$$

where $y_{0}=1-e^{-1}+x e^{-1}$ and $f_{k}$ is the $k$ th iterate of the Poisson generating function

$$
f(y)=\exp (y-1) .
$$

THEOREM 5. $P\left(d\left(T_{n}\right) \geqslant k\right) \rightarrow \pi_{k}$ as $n \rightarrow x$ where

$$
\pi_{k}=\exp \left(\sum_{i=1}^{k-1} \alpha_{i}\right)
$$

and the $x_{i}$ are given recursively by

$$
x_{0}=0, \quad \alpha_{i+1}=\exp \left(\alpha_{i}\right)-e^{-1}-1 .
$$

It is not difficult to show that $x_{i}+1 \sim A e^{-i}$ for some $A>0$. 
ProOF OF THEOREM 3. We show that the joint distribution of the family sizes of members of the $k$ th generation of the branching-type process, conditional upon the past, is the limit as $n \rightarrow \infty$ of the joint distribution of the corresponding quantities associated with the vertices in the $k$ th stratum of $T_{n}$, conditional upon the numbers of vertices in earlier strata.

First consider the process $\left\{Y_{i}: i \geqslant 0\right\}$. List the family sizes $G_{1}, \ldots, G_{r}$ of the $r$ members of the $k$ th generation in some arbitrary order. Their joint probability mass function is

$$
\begin{aligned}
p\left(a_{1}, a_{2}, \ldots, a_{r}\right) & =\sum_{i=1}^{r} a_{i}\left(r e^{r} a_{1} ! a_{2} ! \ldots a_{r} !\right)^{-1} \\
& =\frac{u}{r} \bar{a}_{1} \frac{e^{-r}}{! \ldots a_{r} !}
\end{aligned}
$$

where $u=a_{1}+a_{2}+\ldots+a_{r}$.

Now let $\mathbf{z}=\left(z_{1}, z_{2}, \ldots, z_{k}\right)$ be a sequence of positive integers, and let $T(\mathbf{z}, n)$ be the number of trees with $n$ labelled vertices such that $Z(i, n)=z_{i}$ for $i=1,2, \ldots, k$. Then putting $r=z_{k}$ and $s=1+z_{1}+z_{2}+\ldots+z_{k}$,

$$
T(\mathbf{z}, n)=C(\mathbf{z}, n) \sum_{\mathbf{b}}\left(\begin{array}{c}
n-s \\
b_{1}-1, b_{2}-1, \ldots, b_{r}-1
\end{array}\right) b_{1}^{b_{1}-2} b_{2}^{b_{2}-2} \ldots b_{r}^{b_{r}-2},
$$

where $\sum_{\mathrm{b}}$ sums over all sequences $\left(b_{1}, b_{2}, \ldots, b_{r}\right)$ with sum $n+r-s$ and $C(\mathbf{z}, n)$ is the number of ways of labelling the $s$ vertices in the first $k$ strata.

But consider the number of trees with $N$ labelled vertices $v_{1}, v_{2}, \ldots, v_{N}$ in which $v_{1}$ has degree $t$. The number of such trees is

$$
\left(\begin{array}{c}
N-1 \\
t
\end{array}\right) \sum_{\mathrm{b}}\left(\begin{array}{c}
N-t-1 \\
b_{1}-1, b_{2}-1, \ldots, b_{t}-1
\end{array}\right) b_{1}^{b_{1}-2} \ldots b_{t^{t_{t}-2}}^{b^{2}} \sim N^{N-2}(e(t-1) !)^{-1}
$$

since the probability that $v_{1}$ has degree $t$ in $T_{N}$ approaches $(e(t-1) !)^{-1}$ as $N \rightarrow x$. Thus

$$
T(\mathbf{z}, n) \sim C(\mathbf{z}, n)(n+r-s+1)^{n-s-1} r e^{-1} .
$$

The number of trees contributing to $T(\mathbf{z}, n)$ which have the property that $\left(a_{1}, a_{2}, \ldots, a_{r}\right)$ is the sequence, arbitrarily ordered, of numbers of pendant vertices from the $r$ vertices in the $k$ th stratum is

$$
T(\mathbf{z}, \mathbf{a}, n)=C(\mathbf{z}, n)\left(\begin{array}{c}
n-s \\
a_{1}, a_{2}, \ldots, a_{r}, a
\end{array}\right) \sum_{b}\left(\begin{array}{c}
n-s-u \\
b_{1}-1, \ldots . h_{u}-1
\end{array}\right) b_{1}^{b_{1}-2} \ldots b_{u}^{b_{u}-2},
$$

where $u=a_{1}+a_{2}+\ldots+a_{r}$ and $a=n-s-u$. From (11).

$$
T(\mathbf{z}, \mathbf{a}, n) \sim C(\mathbf{z}, n) \frac{(n-s+1)^{n-s-1}}{a_{1} ! \ldots a_{r} !} u e^{-1} .
$$


Divide (13) by (12) and let $n \rightarrow \infty$ to obtain

$$
\begin{aligned}
T(\mathbf{z}, \mathbf{a}, n) / T(\mathbf{z}, n) & \sim \frac{u}{r} \frac{\left(1+r(n-s+1)^{-1}\right)^{-n}}{a_{1} ! \ldots a_{r} !} \\
& \rightarrow \frac{u}{r} \frac{e^{-r}}{a_{1} ! \ldots a_{r} !}
\end{aligned}
$$

which coincides with (10) as required. This proves the theorem.

ProOF OF THeOREM 4. From (7), the number $Z(k, n)$ in the $k$ th stratum of $T_{n}$ has the same distribution as $X_{k}$. conditional upon $\{N=n\}$. Hence, from (8),

$$
P(Z(k, n)=i) \rightarrow i P\left(X_{k}=i\right) .
$$

Thus, the probability generating function $F_{k, n}(y)=E\left(y^{Z(k, n)}\right)$ satisfies

$$
\begin{aligned}
F_{k, n}(y) & =\sum_{i} y^{i} P(Z(k, n)=i) \\
& \rightarrow \sum_{i} y^{i} i P\left(X_{k}=i\right) \\
& =y \frac{d}{d y} f_{k}(y)=F_{k}(y) .
\end{aligned}
$$

Of course, $Y_{k}$ also has generating function $F_{k}(y)$. Furthermore, the number $N_{k}$ of contributors to $Y_{k}$ which have family size zero is the sum of $Y_{k}-1$ Bernoulli variables, each taking the value 1 with probability $e^{-1}$; the $Y_{k}$ th family is that with the compulsory supplementary member. Hence

$$
\begin{aligned}
H_{k, n}(x) & \rightarrow E\left(x^{N_{k}}\right) \\
& =\left.y^{-1} F_{k}(y)\right|_{y=y_{0}}
\end{aligned}
$$

as required.

PrOOF OF THEOREM 5. Let $\mathbf{i}=\left(i_{1}, i_{2}, \ldots, i_{k}\right)$ be a sequence of positive integers, and let

$$
A_{j}=\left\{N_{j}=0\right\} \quad \text { and } \quad B_{j}=\left\{Y_{j}=i_{j}\right\} \quad \text { for } j=1,2, \ldots, k .
$$

From Theorem 3, $P\left(d\left(T_{n}\right) \geqslant k+1\right) \rightarrow P\left(A_{1} A_{2} \ldots A_{k}\right)$. But, by elementary considerations about conditional probabilities,

$$
P\left(A_{1} A_{2}, \ldots A_{k}\right)=\sum_{\mathrm{i}} \prod_{j=1}^{k} P\left(A_{j} \mid A_{1} \ldots A_{j-1} B_{1} \ldots B_{j}\right) P\left(B_{j} \mid A_{1} \ldots A_{j-1} B_{1} \ldots B_{j-1}\right),
$$

with the convention that the intersection of an empty set of events is the whole sample space. Using the Markov property

$$
\begin{aligned}
P\left(A_{1} A_{2} \ldots A_{k}\right) & =\sum_{\mathrm{i}} \prod_{j=1}^{k} P\left(A_{j} \mid B_{j}\right) P\left(B_{j} \mid A_{j-1} B_{j-1}\right) \\
& =\sum_{\mathrm{i}} \prod_{j=1}^{k}\left(1-e^{-1}\right)^{i_{j}-1} C_{j}\left(i_{j}\right),
\end{aligned}
$$


where $C_{j}(i)$ is the coefficient of $x^{i}$ in the generating function $D_{j}(x)$ of $Y_{j}$ conditional upon $Y_{j-1}=i_{j-1}$ and $N_{j-1}=0$. Thus

$$
Y_{j}=1+P+F_{1}+\ldots+F_{i_{j}-1} \text {. }
$$

where $P$ has the Poisson distribution and the $F_{i}$ are independent variables each with the Poisson distribution conditioned on being nonzero. Hence

$$
D_{j}(x)=x e^{x-1}\left(\left(e^{x}-1\right) /(e-1)\right)^{i_{j}},-1 .
$$

Summing (14) over $i_{k}$ gives

$$
P\left(A_{1} A_{2} \ldots A_{k}\right)=\sum_{\left(i_{1}, \ldots, i_{k-1}\right)} \prod_{j=1}^{k-1} \beta_{1}^{i,-1} C_{j}\left(i_{j}\right) e^{\beta_{1}} \quad 1\left(\frac{e^{\beta_{1}}-1}{e-1}\right)^{i_{k}-1},
$$

where $\beta_{1}=1-e^{-1}$. Sum (15) over $i_{k-1}$ to obtain

$$
P\left(A_{1} A_{2} \ldots A_{k}\right)=\sum_{\left(i_{1} \ldots, \ldots, i_{k-2}\right)} \prod_{j=1}^{k-2} \beta_{1}^{i_{j}-1} C_{j}\left(i_{j}\right) e^{\beta_{1}-\beta_{2}-2}\left(\frac{e^{\beta_{2}}-1}{e-1}\right)^{i_{k}-1}
$$

where $\beta_{2}=e^{-1}\left(\exp \left(\beta_{1}\right)-1\right)$. Continue, to show that

$$
P\left(A_{1} A_{2} \ldots A_{k}\right)=\exp \left(\sum_{i=1}^{k}\left(\beta_{i}-1\right)\right)=\exp \left(\sum_{i=1}^{k} x_{i}\right) \text { for } k \geqslant 1,
$$

as required, where $\beta_{0}, \beta_{1}, \ldots$ are given by the recursion

$$
\beta_{0}=1, \quad \beta_{i+1}=e^{-1}\left(\exp \left(\beta_{i}\right)-1\right),
$$

and $\alpha_{i}=\beta_{i}-1$. It is easy to check that the $\beta_{i}$ satisfy $\beta_{i} \sim A e^{-i}$ for some $A>0$.

\section{Acknowledgement}

I am very grateful to Professor J. W. Moon for indicating and correcting an error in an earlier combinatorial argument which I used in Section 2.

\section{References}

G. R. Grimmett (1980), 'Random graphs', Further selected to pics in yruph theory, edited by L. Beineke and R. Wilson (Academic Press), to appear.

D. P. Kennedy (1975), 'The Galton-Watson process conditioned on the total progeny', J. Appl. Probability 12, 800-806.

V. F. Kolchin (1977), 'Branching processes, random trees, and a generalized scheme of arrangements of particles', Math. Notes 21, 386-394.

A. Meir and J. W. Moon (1970), 'The distance between points in random trees'. J. Combinatorial Theory 8. 99-103.

A. Meir and J. W. Moon (1975), 'Climbing certain types of rooted trees I. Proc. 5th British Comhinatorial Conf., pp. $461-469$. 
A. Meir and J. W. Moon (1978a). Climbing certain types of rooted trees Il, Acta Math. Acad. Sci. Hungar. 31. 4354.

A. Meir and J. W. Moon (1978b). ( On the altitude of nodes in random trees', Canad. J. Math.30.997-1015.

J. W. Moon (1970). 'Climbing random trees'. Aequationes Math. 5. 68-74.

J. W. Moon (1973), 'Random walks on random trees', J. Austral. Math. Soc. 15, 42-53.

A. Rényi and G. Szekeres (1967). 'On the height of trees', J. Austral. Math. Soc. 7, 497-507.

V. E. Stepanov (1969), 'On the distribution of the number of vertices in strata of a random tree', Theor. Probability Appl. 14, 65-78.

School of Mathematics

University of Bristol

Bristol BS8 1TW

England 